\title{
Designing coupled free-form surfaces
}

\author{
R. Andrew Hicks ${ }^{1, *}$ and Christopher Croke $^{2}$ \\ ${ }^{1}$ Department of Mathematics, Drexel University \\ 3141 Chestnut Street, Philadelphia, PA 19104, USA \\ ${ }^{2}$ Department of Mathematics, University of Pennsylvania \\ 209 South 33rd Street, Philadelphia, PA 19104, USA \\ ahicks@math.drexel.edu
}

The problem of designing optical systems that contain free-form surfaces is a challenging one, even in the case of designing a single surface. Here we present a method for the coupled design of two free-form reflective surfaces which will have a prescribed distortion. On one hand, the method can be described using traditional vectors and matrices, which we do, but it is motivated by viewing the problem in the language of distributions from differential geometry and makes use of the exterior differential systems, which we relegate to an appendix. Example applications are given to the design of a mirror pair that increases the field of view of an observer, a similar mirror pair that also rotate the observers view, and a pair of mirrors that give the observer a traditional panoramic strip view of the scene. (c) 2010 Optical Society of America

OCIS codes: $080.2740,110.0110$, , 230.4040.

\section{Introduction}

The problem that we consider here can be simply stated: how does one design a pair of reflectors so that when viewed with the human eye or pinhole camera, the image of a given object plane (or surface more generally) appears with a prescribed distortion to the observer. One can think of this as designing a periscope with curved mirrors to achieve a prescribed purpose such as having wide angle of view. This problem was considered in $[1,2]$ by the first author and R. Perline. An example of this problem in which only one reflector is used is the design of a driver-side mirror for a motor vehicle, which has no blind-spot and yet provides the observer with a perspective view of the scene [3]. Unfortunately though, like most optics problems, more than one reflector is required to solve this problem. Here we show that the problem is solvable using a pair of reflectors, and we present an algorithm for constructing 
these reflectors. Although this paper is written with a pair of mirrors in mind, everything also works with a pair of lenses instead. Since a given piece of glass has two surfaces to design, we could design such optical systems with a single piece of glass. It is also straightforward to extend the method to more than two optical elements.

Let us first consider the problem of constructing a single reflecting surface. It is convenient to view the pinhole as a source from which rays emanate (by the reversibility of geometric optics). An object surface $S$ is to be imaged onto a plane $I$ in a prescribed way. In other words a tranformation $\mathbf{F}: I \rightarrow S$ is part of the data of the problem, as in Fig. 1. This mapping corresponds to controlling the distortion of the mirror. Given such a correspondence, we note that a vector field $\mathbf{W}$ is then defined on some subset of $\mathbb{R}^{3}$ via the construction given in Fig. 1. If a solution surface $M$ exists then $\mathbf{W}$ will be perpendicular to it. Of course, the length of $\mathbf{W}$ is irrelevant - our goal is to find a surface that will be perpendicular to $\mathbf{W}$.

It is very unlikely, but if $\nabla \times \mathbf{W}=0$ then $\mathbf{W}=\nabla \phi$ for some $\phi$ and the level surfaces

$$
\phi(x, y, z)=\text { constant }
$$

are each a solution to the problem. It may be that $\mathbf{W}$ is not a gradient, but a multiple of a gradient, which occurs if and only $(\nabla \times \mathbf{W}) \cdot \mathbf{W}=0$ (See [4] or [5]).

Solutions to such problems naturally tend to consist of free-form surfaces, i.e. surfaces that lack rotational symmetry. An early example of an optical free-form surface is contained in patent by Kanolt for a progressive lens [6]. A familiar, and commercially successful product that used free-form surfaces is the Polaroid SX-70 camera [7]. Stone and Forbes studied the first-order properties of such systems $[8,9]$, but comparatively little theory exists otherwise. The likely reason for this is that until recently, the grinding of such surfaces was extremely difficult. In approximately the last ten years though, with technology developed by the DARPA conformal optics program [10], techniques, such as raster grinding have become commercially available [11]. It would appear that free-form surfaces could play a role in numerous applications that by their nature lack rotational symmetry, but methods for the design of free-form surfaces are in their infancy. Illumination is a natural application area, and overviews include the book by Winston et al [12] and the article by Minano et al [13]. For illumination, even the problem of controlling the intensity from a single point source with a single reflector is quite hard. It was solved theoretically and numerically by Oliker and Kochengin in $[14,15]$.

The design of multiple free-form surface systems is considerably more complicated. For illumination the problem has been studied extensively Benitez et al starting in [16]. Applications to laser beam shaping have been considered by Rubinstein and Wolansky in [17], who consider a coupled pair of free-form lenses. Two reflector systems for illumination are investigated Glimm and Oliker in [18]. 


\section{The Design Algorithm}

The two-mirror case may be modeled with a system of partial differential equations. Consider the problem of building a two mirror system to realize a given correspondence $\mathbf{F}$ between the image plane $x=1$ and the object surface. We consider the rays to be emanating from a source $L$ at the origin (or entering a pinhole there). We consider $L$ as the eye of an observer which is looking down the $\mathrm{x}$-axis, in the positive direction. As in Fig. 2, a ray traveling from $L$ through a point $A=(1, s, t)$ is to travel to $\mathbf{F}(s, t)$ by reflecting off of two mirrors at the points $\mathbf{P}$ and $\mathbf{Q}$. This requirement determines a pair of normals, $\mathbf{W}$ and $\mathbf{V}$, at $\mathbf{P}(A)$ and $\mathbf{Q}(A)$ respectively. Thus we seek two mirrors, parameterized as

$$
\mathbf{P}:(s, t) \mapsto(x(s, t), y(s, t), z(s, t)),
$$

and

$$
\mathbf{Q}:(s, t) \mapsto(u(s, t), v(s, t), w(s, t))
$$

where to ensure that the light ray reflects from $P$ to $Q$ to $\mathbf{F}(A)$ we demand that the tangent vectors to the above surfaces be perpendicular to the vectors $\mathbf{W}$ and $\mathbf{V}$. This gives a system of four partial differential equations in four unknowns:

$$
\begin{gathered}
\mathbf{P}_{s}(s, t) \cdot \mathbf{W}(s, t)=0, \mathbf{P}_{t}(s, t) \cdot \mathbf{W}(s, t)=0 \\
\mathbf{Q}_{s}(s, t) \cdot \mathbf{V}(s, t)=0, \mathbf{Q}_{t}(s, t) \cdot \mathbf{V}(s, t)=0 .
\end{gathered}
$$

$\mathbf{W}$ and $\mathbf{V}$ are computed by finding the unit directions from various points and adding them.

$$
\begin{gathered}
\mathbf{W}=\frac{(0,0,0)-\mathbf{P}(s, t)}{|(0,0,0)-\mathbf{P}(s, t)|}+\frac{\mathbf{Q}(s, t)-\mathbf{P}(s, t)}{|\mathbf{Q}(s, t)-\mathbf{P}(s, t)|} . \\
\mathbf{V}=\frac{\mathbf{P}(s, t)-\mathbf{Q}(s, t)}{|\mathbf{P}(s, t)-\mathbf{Q}(s, t)|}+\frac{\mathbf{F}(s, t)-\mathbf{Q}(s, t)}{|\mathbf{F}(s, t)-\mathbf{Q}(s, t)|} .
\end{gathered}
$$

An abstract, but useful way to think of the problem is to then view the two parameterized surfaces as a single parameterized surface in six dimensional space $\mathbb{R}^{6}$, with the parametrization

$$
\Gamma(s, t)=(x(s, t), y(s, t), z(s, t), u(s, t), v(s, t), w(s, t)) .
$$

We emphasize that this is still a two dimensional object, and so does have a two dimensional tangent plane at each point, which is spanned by $\boldsymbol{\Gamma}_{s}$ and $\boldsymbol{\Gamma}_{t}$. One advantage of this way of looking at things is that the above equations can be interpreted as saying that for all $(s, t)$ both $\boldsymbol{\Gamma}_{s}$ and $\boldsymbol{\Gamma}_{t}$ are perpendicular to two vector fields $\widetilde{\mathbf{W}}$ and $\widetilde{\mathbf{V}}$ in $\mathbb{R}^{6}$. To define $\widetilde{\mathbf{W}}$ and $\widetilde{\mathbf{V}}$, for each 6 -tuple $(x, y, z, u, v, w)$ the vector $\left(W_{1}, W_{2}, W_{3}\right)$ in $\mathbb{R}^{3}$ is defined by 


$$
\frac{(0,0,0)-(x, y, z)}{|(0,0,0)-(x, y, z)|}+\frac{(u, v, w)-(x, y, z)}{|(u, v, w)-(x, y, z)|},
$$

and then we define the vector field $\widetilde{\mathbf{W}}$ in $\mathbb{R}^{6}$ as

$$
\widetilde{\mathbf{W}}(x, y, z, u, v, w)=\left(W_{1}, W_{2}, W_{3}, 0,0,0\right) .
$$

Similarly

$$
\widetilde{\mathbf{V}}(x, y, z, u, v, w)=\left(0,0,0, V_{1}, V_{2}, V_{3}\right) .
$$

where $\left(V_{1}, V_{2}, V_{3}\right)$ is the three dimensional vector

$$
\frac{(x, y, z)-(u, v, w)}{|(x, y, z)-(u, v, w)|}+\frac{\mathbf{F}(y / x, z / x)-(u, v, w)}{|\mathbf{F}(y / x, z / x)-(u, v, w)|} .
$$

Note that in the above $\mathbf{F}(y / x, z / x)$ geometrically represents projecting the point $(x, y, z)$ onto the image plane $x=1$, and then applying $T$ to it.

We propose to find a solution $\Gamma(s, t)$ that contains an initial curve

$$
\mathbf{C}(t)=\left(C_{1}(t), C_{2}(t), C_{3}(t), C_{4}(t), C_{5}(t), C_{6}(t)\right)
$$

which is assumed to be perpendicular to $\widetilde{\mathbf{V}}$ and $\widetilde{\mathbf{W}}$. There are many ways to find such initial curves which usually amounts to solving an ordinary differential equation. We give explicit examples in the next section. The first three components of the curve, $\left(C_{1}(t), C_{2}(t), C_{3}(t)\right.$ defines a curve $C_{L}(t)$ in one of the mirrors (the "lower mirror"), and the second three, $\left(C_{4}(t), C_{5}(t), C_{6}(t)\right)$, defines a curve $C_{U}(t)$ in the other mirror (the "upper mirror").

In sharp contrast to the case of a single mirror (where no solution is expected), in general one can expect that there exists a unique solution surface (albeit possibly very small) passing through the curve $\mathbf{C}(t)$. See the appendix for a more precise statement.

The idea for how to compute this solution is to "grow" a solution surface out from $\mathbf{C}(t)$ by computing the tangent spaces at each point along it, and approximating a thin strip of the solution surface by using the tangent planes. The technical difficulty then is how one could compute a tangent plane to $\Gamma$ along the curve, assuming that the curve lies in a solution surface $\boldsymbol{\Gamma}$. Choosing a point on the curve, $\mathbf{C}\left(t_{0}\right)$, we know one of the vectors that span the tangent plane, namely $\mathbf{C}^{\prime}\left(t_{0}\right)$. How do we find the entire tangent space? The space of candidate vectors is 6 dimensional. We will show how to produce four linear equations that will (unless things are degenerate) reduce the dimension of choices to two, and thus determine our tangent space.

We already know two of the equations. If $\mathbf{T}$ is a tangent vector to $\boldsymbol{\Gamma}$ at $\mathbf{C}\left(t_{0}\right)$ it must satisfy the two linear equations

$$
\widetilde{\mathbf{W}}\left(\mathbf{C}\left(t_{0}\right)\right) \cdot \mathbf{T}=0
$$


and

$$
\widetilde{\mathbf{V}}\left(\mathbf{C}\left(t_{0}\right)\right) \cdot \mathbf{T}=0
$$

There are two more equations. We define 6-by-6 matrices $A=A\left(\mathbf{C}\left(t_{0}\right)\right)$ and $B=B\left(\mathbf{C}\left(t_{0}\right)\right)$ by the equations

$$
A_{i, j}=\frac{\partial \widetilde{\mathbf{W}}_{i}}{\partial x_{j}}-\frac{\partial \widetilde{\mathbf{W}}_{j}}{\partial x_{i}}
$$

and

$$
B_{i, j}=\frac{\partial \tilde{\mathbf{V}}_{i}}{\partial x_{j}}-\frac{\partial \tilde{\mathbf{V}}_{j}}{\partial x_{i}} .
$$

It is a fact (see the appendix) that if $\mathbf{S}$ and $\mathbf{T}$ are tangent (column) vectors to our solution surface, then $A$ and $B$ annihilate them in the sense that

$$
\mathbf{S}^{t} A \mathbf{T}=0, \mathbf{S}^{t} B \mathbf{T}=0 .
$$

Since $\mathbf{C}^{\prime}\left(t_{0}\right)$ is tangent to $\boldsymbol{\Gamma}$ we can use it for $\mathbf{S}$ above. Inserting that vector into the above gives two additional linear equations for a total of four linear equations for $\mathbf{T}$ at $\mathbf{C}\left(t_{0}\right)$ :

$$
\widetilde{\mathbf{W}} \cdot \mathbf{T}=0, \quad \widetilde{\mathbf{V}} \cdot \mathbf{T}=0, \quad \mathbf{C}^{\prime t} A \mathbf{T}=0, \quad \mathbf{C}^{\prime t} B \mathbf{T}=0 .
$$

This describes the full two dimensional space of tangent vectors $\mathbf{T}$.

The initial curve $\mathbf{C}^{0}(t)=\mathbf{C}(t)$ must of course be discretized as a finite (ordered) collection of points in $\mathbb{R}^{6}$. At each of these points $p$, we may compute the tangent space of the solution surface by using the four linear equations (19). As in Fig. 3 one "pushes off", by choosing a tangent vector $w$ at each $p$, to form a new discretized curve, $\mathbf{C}^{1}(t)$ (i.e. the point on the new curve is $p+w)$. This pushing off may be done in an infinite number of directions $w$. For example, one could demand that $w$ be perpendicular to $\mathbf{C}^{\prime}(p)$ ( $\mathbf{V}$ in figure 3 ) and $|w|$ is some small step size. However, in practice there are (for numerical reasons) often better choices for $w$. The construction should lead to a new curve $\mathbf{C}^{1}$ which is an approximate solution curve (i.e. is approximately perpendicular to $\widetilde{\mathbf{V}}$ and $\widetilde{\mathbf{W}}$ ) and thus we can continue the process to find a new curve $\mathbf{C}^{2}$ from $\mathbf{C}^{1}$ etc. generating our solution surface in six dimensions. This surface in turn describes the two mirrors in three space that give a solution to our problem.

\section{Examples}

In this section we give some examples. Once we are given the desired transformation $T$ (which defines our vector fields $\widetilde{\mathbf{V}}$ and $\widetilde{\mathbf{W}}$ ) there are three steps. The first is to choose an 
initial curve $\mathbf{C}(t)$ everywhere perpendicular to $\widetilde{\mathbf{V}}$ and $\widetilde{\mathbf{W}}$. Next we apply the method to yield two mirrors and finally we test the result with a ray tracing program.

There are many ways to choose the initial curve $\mathbf{C}(t)$ and the best choice will depend on the application in mind. There is however a nice choice of curves that will give nice mirrors in a number of cases. We choose $\mathbf{C}(t)$, for $t$ in an interval about 0 , of the form

$$
\mathbf{C}(t)=(9 \cos (t), 9 \sin (t), 0,9 \cos (t), 9 \sin (t), \beta(t)) .
$$

That is the lower curve will be an arc of the circle of radius 9 in the $x-y$ plane. The upper curve will lie directly above the lower curve but have variable height. (Of course 9 is arbitrary and is chosen depending on where you want your lower mirror to be.) An advantage of these curves is that whatever $T$ is or your choice of $\beta(t)$ these curves will be perpendicular to $\widetilde{\mathbf{W}}$. Thus we only need to choose $\beta(t))$ so that $\mathbf{C}^{\prime}(t)$ is perpendicular to $\widetilde{\mathbf{V}}(\mathbf{C}(t))$. This leads to a first order ODE for $\beta(t)$ which is easy to solve (sometimes even in closed form - depending on $T$ ) given an initial condition (e.g. an initial height the upper mirror will be above the lower one).

There are a number of ways to implement the method. The choice basically amounts to how to choose the $w$ at each step. It is also often useful to parameterize each new curve by arclength. In this paper we wont go into the specific choices we made in the examples below.

We tested our mirrors using the program POV-Ray. This is a ray tracing program. We put our mirrors in a cubical room with checkerboard walls. The wall we are looking towards is the "x-positive" wall. It consists of red and black squares and has the words "this is $\mathrm{x}$ positive" in blue. The view point is the origin and we will be looking at the lower mirror. (The upper mirror is out of our field of view.) Behind the mirror is the wall which we see directly.

The first example will be a "wide angle periscope" with initial curve described above. The expansion factor is four. Figure 4 gives a view of the two mirrors as generated by Maple. Figure 5 gives the view (via ray tracing) of the room described above. Here the field of view of the observer subtended by the mirror is approximately $12^{\circ}$ and the resulting field of view in the mirror is approximately $33^{\circ}$.

The second example not only has the wide angle (with a factor of 4 ) but a rotation of $45^{\circ}$. Figure 6 shows the view of the room and Fig. 7 shows the view of the room in the mirror.

For the third example we aimed for a $360^{\circ}$ panoramic view. The mirrors will take $360^{\circ}$ and image it in $360^{\circ} / 12=30^{\circ}$. The vertical is scaled by a factor of 16 to make the horizontal and vertical scalings similar and to include a view of part of the floor. Figure 8 shows the mirror pair while Figure 9 shows the view of the room. In this case we see more than the front wall. The wall to the left is a black and green checkerboard, the one to the right green and white, the wall behind you is red and white, while the floor is blue and white. Note that 
we viewing the room with a wider angle than in the other examples.

In the fourth example we revisit the first example, i.e. a wide angle periscope with magnification factor of 4 . However, this time we use a different initial curve $\mathbf{C}(t)$. Here the lower curve $\mathbf{C}_{L}(t)$ is a straight line in the plane $y=0$ the upper curve also lies in this plane. Figure 10 shows the mirrors and figure 11 the room. It is interesting to compare figures 10 and 4 to see two different solutions to the same problem (due to different initial curves).

(We should point out that in the fourth example there is a difficulty. This because our initial curve has both upper and lower parts lying in the plane $y=0$ - which is a plane of symmetry. It turns out that the four linear equations (19) are degenerate in this case. We overcame this by having the initial curves be very slightly off this plane. This allowed the procedure to start, and since the generated curves are sufficiently far away from $y=0$ we got a nice solution.)

\section{Appendix - The differential forms viewpoint}

To phrase the problem in terms of differential forms, we note that dual to $\widetilde{\mathbf{W}}$ and $\widetilde{\mathbf{V}}$ are one forms $\theta_{1}$ and $\theta_{2}$ (e.g. for a tangent vector $\mathbf{X} \in \mathbb{R}^{6}$ we have $\theta_{1}(\mathbf{X})=\widetilde{\mathbf{W}} \cdot \mathbf{X}$ ). The common kernel (i.e. the vectors that are simultaneously perpendicular to $\widetilde{\mathbf{W}}$ and $\widetilde{\mathbf{V}}$ ) is a four-dimensional distribution $E$ in $\mathbb{R}^{6}$. This means that for each $p \in \mathbb{R}^{6}$ there is a four dimensional subspace $E_{p}$ of tangent vectors. We seek a 2 dimensional integral surface, $\boldsymbol{\Gamma}$ (i.e. a surface which at every point $p$ has its tangent vectors in $E_{p}$ ). To construct a solution surface using finite difference methods for example, one would like to find the tangent space at a given point, but the equations given by $\theta_{i}(\mathbf{T})=0$ are not sufficient. We will see that, additional equations must hold that provide this information. Namely, it must be the case that $d \theta_{1}$ and $d \theta_{2}$ must also vanish on the tangent bundle of any solution. This is formalized as follows.

In general, suppose that $E$ is a 4-distribution on a 6-manifold $M$, which is the is the kernel of a pair of 1-forms, say $\theta_{1}$ and $\theta_{2}$. Say that a nonzero vector $\mathbf{V}$ in $E_{p}$ is Kähler-regular if the set of 4 linear equations

$$
\theta_{1}(\mathbf{W})=\theta_{2}(\mathbf{W})=d \theta_{1}(\mathbf{V}, \mathbf{W})=d \theta_{2}(\mathbf{V}, \mathbf{W})=0
$$

for $\mathbf{W}$ in $T_{p} M$ has maximum rank, four, which would result in a two-dimensional solution. An important technical fact that we are using here is that if a form $\psi$ vanishes on an integral surface, then so will the form $d \psi$. (See page 11 of [19].) The idea is that these four equations will determine a unique tangent space to a solution surface at $p$, if $\mathbf{V}$ is given. Similarly, say that an immersed curve $\mathbf{C}$ in $M$ is Kähler-regular if its tangent vectors are Kähler-regular at each point of $\mathbf{C}$. (Note that, in particular, such a curve $\mathbf{C}$ is a 1 dimensional integral of the distribution E.) In this case, the Cartan-Kähler theorem then says that if $\mathrm{E}$ is real-analytic 
and $\mathbf{C}$ is a real-analytic, Kähler-regular integral curve of $E$, then $\mathbf{C}$ lies in a locally unique real-analytic 2 dimensional integral surface of $E$ (see [19]). In other words, generically, if the initial data is tangent to the distribution, then a unique solution surface will exist that contains it.

Thus, in some generic sense, given a 4-distribution $E$ on a 6 -manifold, the generic vector $\mathbf{V}$ in $E_{p}$ (for $p$ a generic point in $M$ ) is Kähler-regular. Thus, for most 4-distributions, one expects existence and uniqueness of 2 dimensional integral surfaces. In the above example of multiple surface design, we take $\theta_{1}$ and $\theta_{2}$ to be the 1 -forms in an open subset of $\mathbb{R}^{6}$ that are dual to the vector fields $\widetilde{\mathbf{W}}$ and $\widetilde{\mathbf{V}}$. In our problems, all of the quantities are real analytic, and in fact often algebraic. Equation (21), which corresponds directly to equation (19) then hints at the method of numerically constructing a solution. Finding an initial curve C amounts to solving an under-constrained differential equation, i.e., every distribution has many 1-dimensional integrals. This is the technique described above for solving the problem.

\section{Acknowledgements}

The authors would like to thank Robert Bryant for useful discussions about this problem. The first author was supported by NSF IIS-04-13012 and NSF DMS-09-08299. The second author was supported by NSF DMS-07-04145 and NSF DMS-10-03679.

\section{References}

1. R. A. Hicks and R. Bajcsy, "Catadioptic sensors that approximate wide-angle perspective projections," in "Proc. Computer Vision Pattern Recognition," (IEEE Computer Society, Los Alamitos, 2000), pp. 545-551.

2. R. Hicks and R. Perline, "The blind-spot problem for motor vehicles," Applied Optics 44, 3893-3897 (2005).

3. R. A. Hicks, "Controlling a ray bundle with a free-form reflector," Opt. Lett. 33, 16721674 (2008).

4. O. Stavroudis, The Mathematics of Geometrical and Physical Optics (Wiley-VCH, Morlenbach, 2006).

5. H. Buchdahl, An Introduction to Hamiltonian Optics (Dover Publications, New York, NY, 1993).

6. C. Kanolt, Multifocal Opthalmic Lenses (United States Patent 2,878,721, March 24th, 1959).

7. W. T. Plummer, "Unusual optics of the polaroid sx-70," Applied Optics 21, 196-202 (1982).

8. B. Stone and G. Forbes, "Foundation of first-order layout for asymmetric systems: an application of hamilton's methods," J. Optical Soc. Amer. A 9, 96-109 (1992). 
9. B. Stone and G. Forbes, "Characterization of first-order optical properties for asymmetric systems," J. Optical Soc. Amer. A 9, 478-489 (1992).

10. E. Kreifeldt, "Darpa turns researchers loose on new class of optics," Optics and photonics News 8, 6-10 (1997).

11. B. Creber, "Fabrication of freeform optics," in "ASPE Proceedings, Free-Form Optics: Design, Fabrication, Metrology, Assembly," , vol. 1 (2004), vol. 1.

12. R. Winston, J. Minano, and P. Benitez, Nonimaging Optics (Elsevier Academic Press, Burlington, Mass., 2005).

13. J. Minano, P. Benitez, and A. Santamaria, "Free-form optics for illumination," Optical Review 16, 99-102 (2009).

14. S. Kochengin and V. Oliker, "Determination of reflector surfaces from near-field scattering data," Numerische Mathematik 79, 553-568 (1998).

15. S. Kochengin and V. Oliker, "Determination of reflector surfaces from near-field scattering data. II. Numerical solution," Numer. Math. 79, 553-568 (1998).

16. P. Gimenez-Benitez, J. C. Miñano, J. Blen, R. Mohedano Arroyo, J. Chaves, O. Dross, M. Hernandez, and W. Falicoff, "Simultaneous multiple surface optical design method in three dimensions," Optical Engineering 43, 1489-1502 (2004).

17. J. Rubinstein and G. Wolansky, "Intensity control with a free-form lens," J. Optical Soc. Amer. A 24, 463-469 (2007).

18. T. Glimm and V. Oliker, "Optical design of two-reflector systems, the monge-kantorovich mass transfer problem and fermat's principle," Indiana Univ. Math. J. 53, 1255-1277 (2004).

19. T. Ivey and J. Landsberg, Cartan for Beginners: Differential Geometry via Moving Frames and Exterior Differential Systems (American Mathematical Society, Providence Rhode Island, 2003). 


\section{List of Figure Captions}

Fig. 1. Given a correspondence, $T$, that points from an image plane to points on a object surface, one can define a vector field $\mathbf{W}$ that is hopefully normal to a mirror surface that realizes the correspondence.

Fig. 3. A two mirror system for controlling a ray bundle emanating from a single source, $L$. Fig. 4 A numerical method for generating a solution surface from an initial Kähler-regular curve.

Fig. 5 Initial Cauchy data for our problem, which lies in a plane, with some sample rays traced.

Fig. 7 A raytracing simulation of the mirror pair. The wall is visible beyond the lower mirror, which presents a wide-angle view of the wall. 


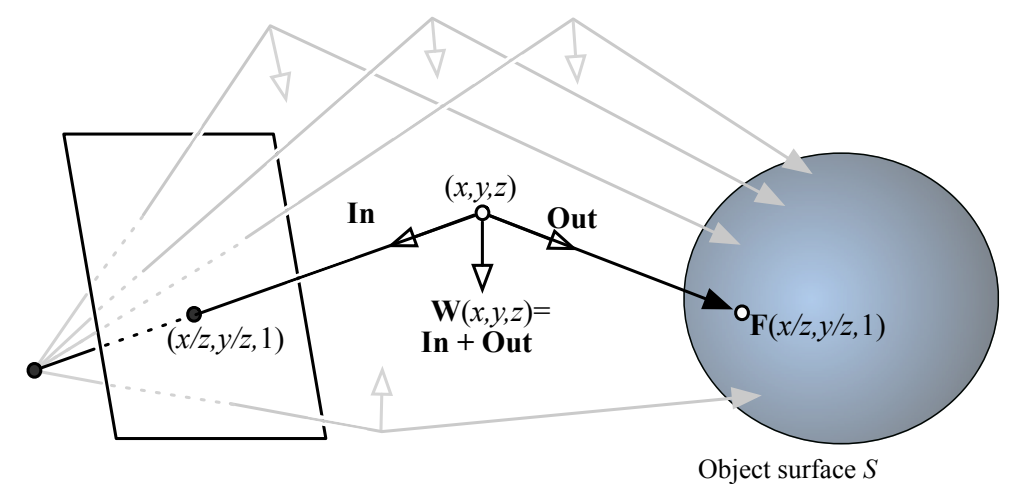

Fig. 1. Given a tranformation, $\mathbf{T}$, that points from an image plane to points on a object surface, one can define a vector field $\mathbf{W}$ that will be normal to any mirror surface that realizes the correspondence. In and Out are unit vectors. 


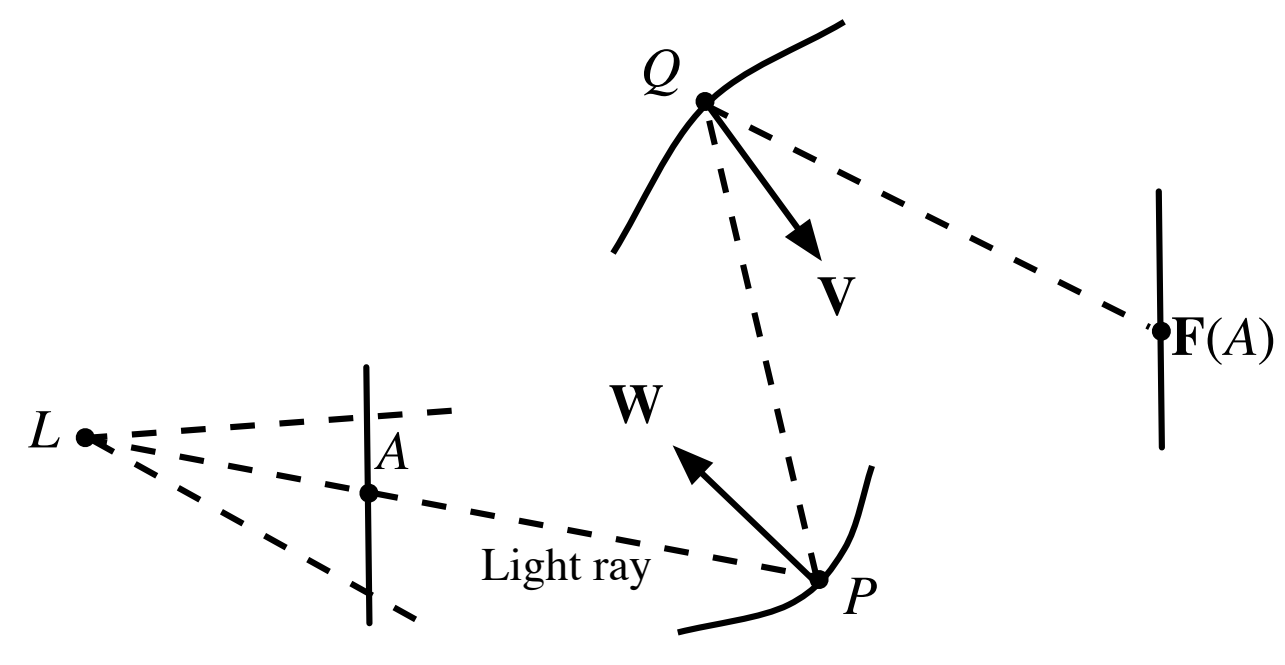

Fig. 2. A two mirror system for controlling a ray bundle emanating from a single source, $L$. Likewise, this could be viewed as a system consisting of a pinhole camera and two reflectors. 


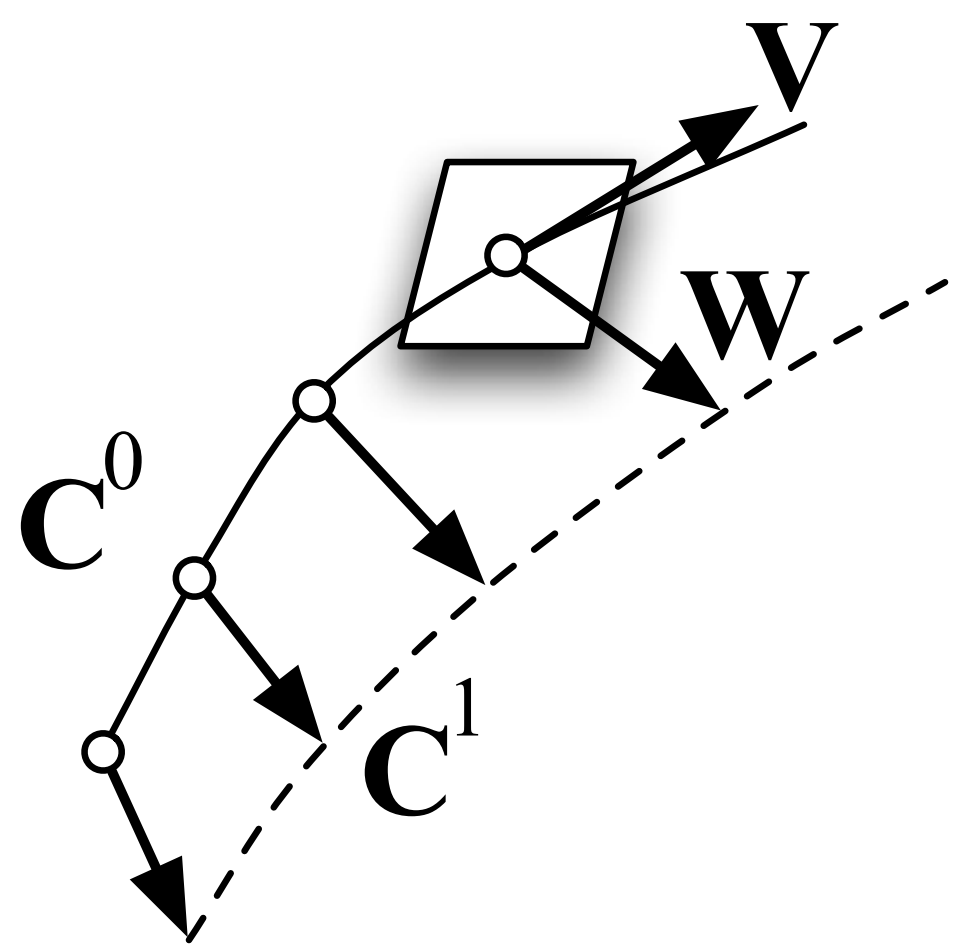

Fig. 3. A numerical method for generating a solution surface from an initial Kähler-regular curve. 


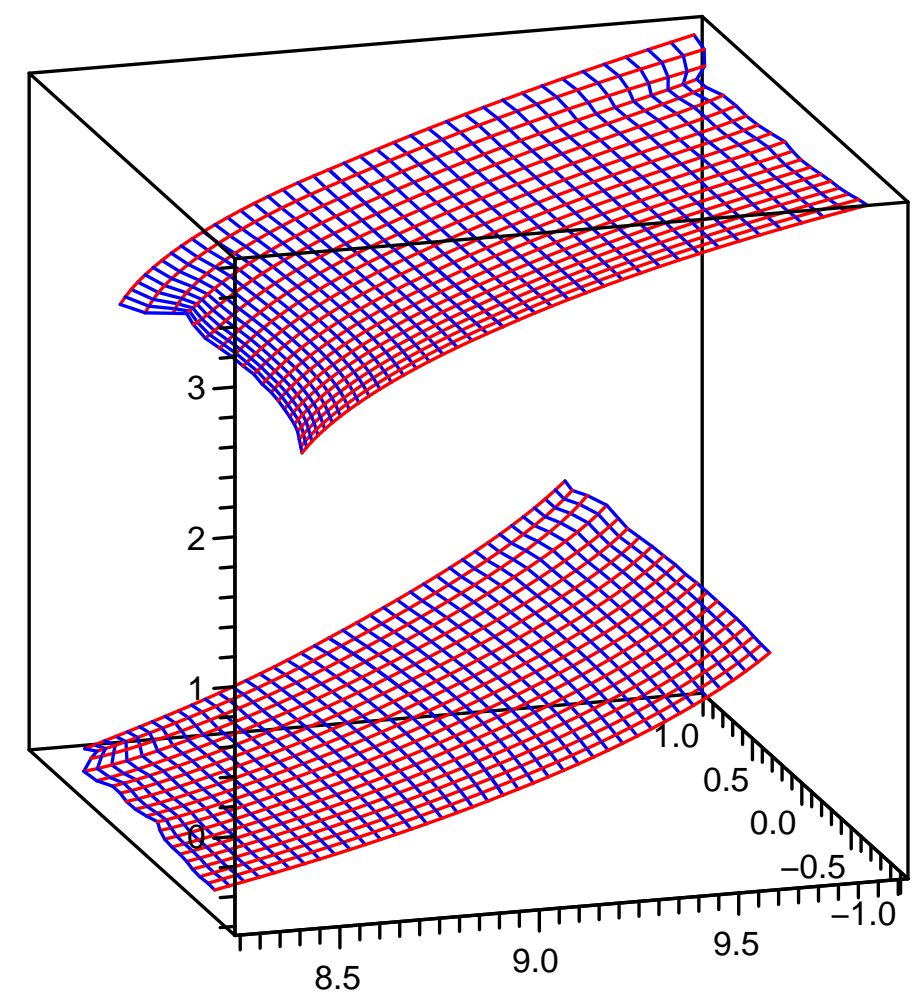

Fig. 4. A pair of mirrors which increases an observers field of view. 


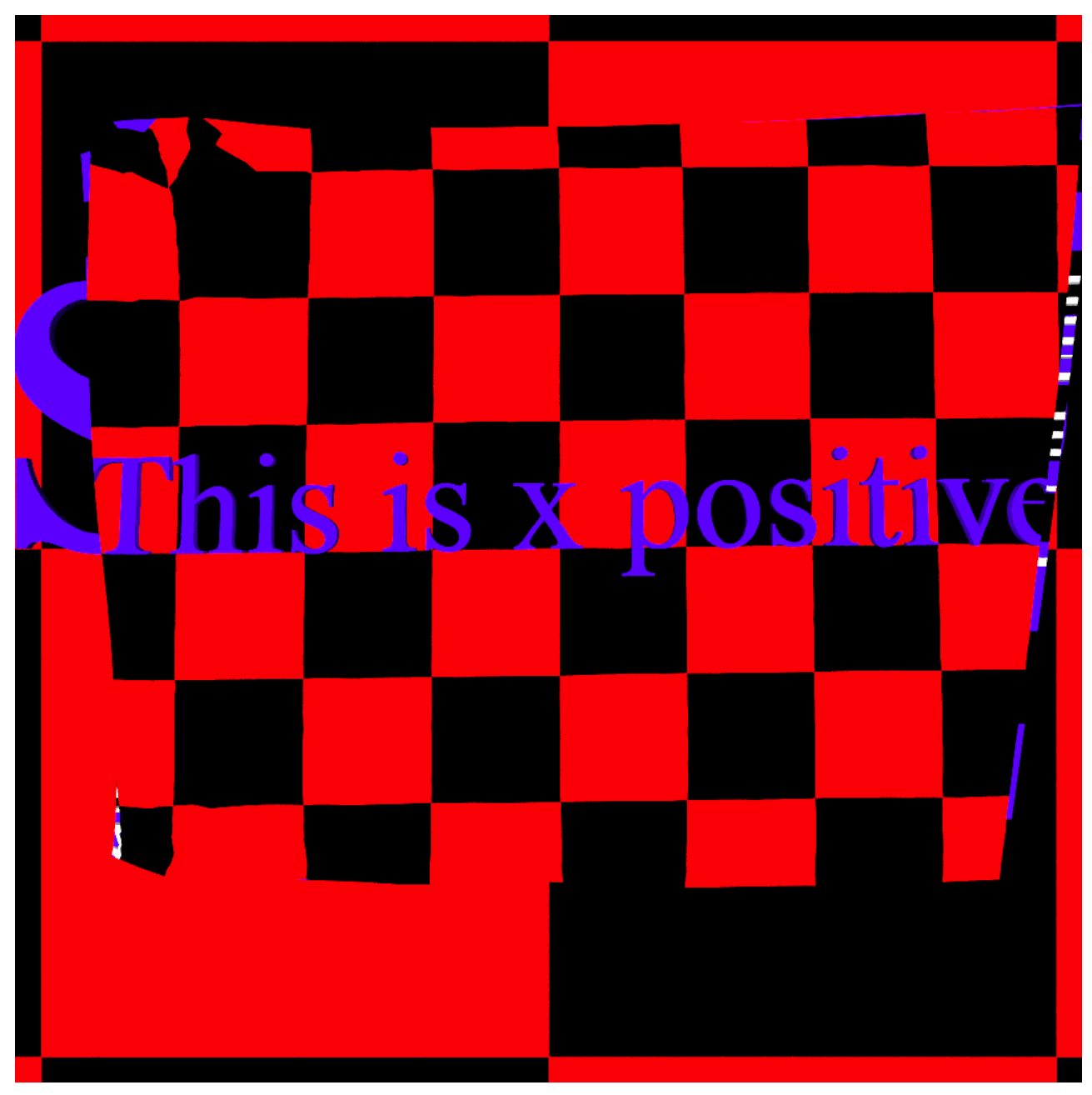

Fig. 5. A raytracing simulation of the mirror pair in Fig. 4. The wall is visible beyond the lower mirror, which presents a wide-angle view of the wall. 


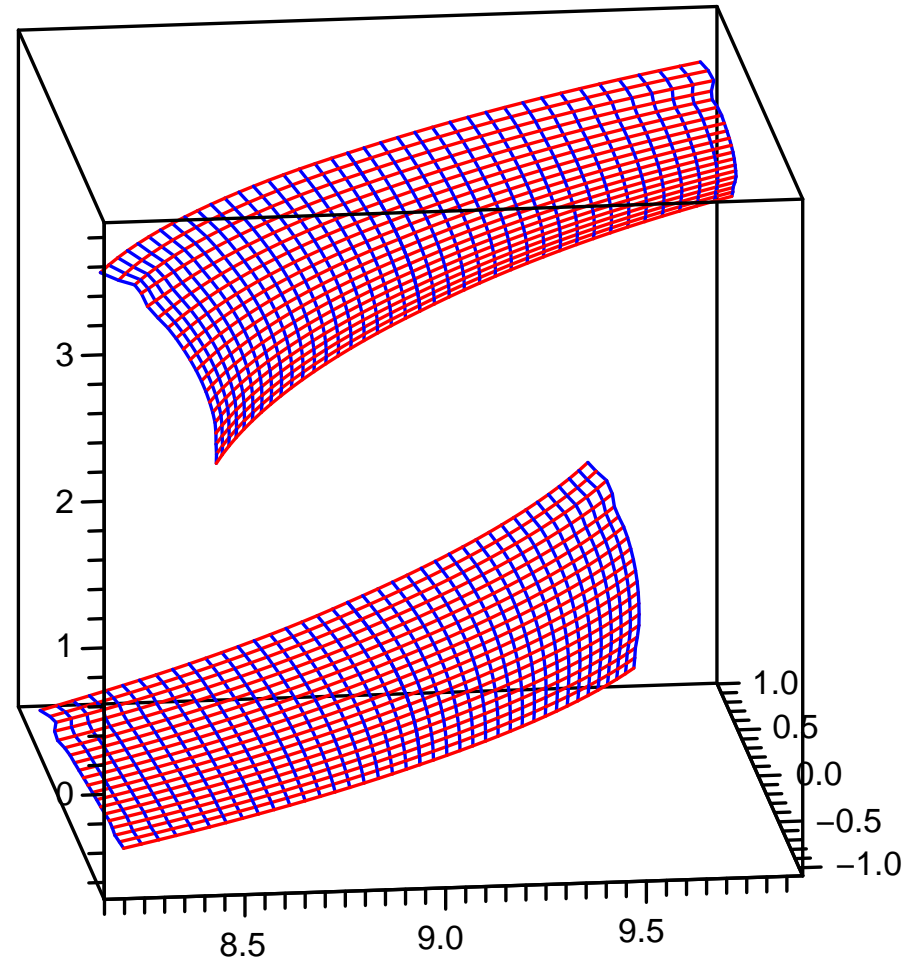

Fig. 6. A wide-angle pair that also rotates the image by $45^{\circ}$. 


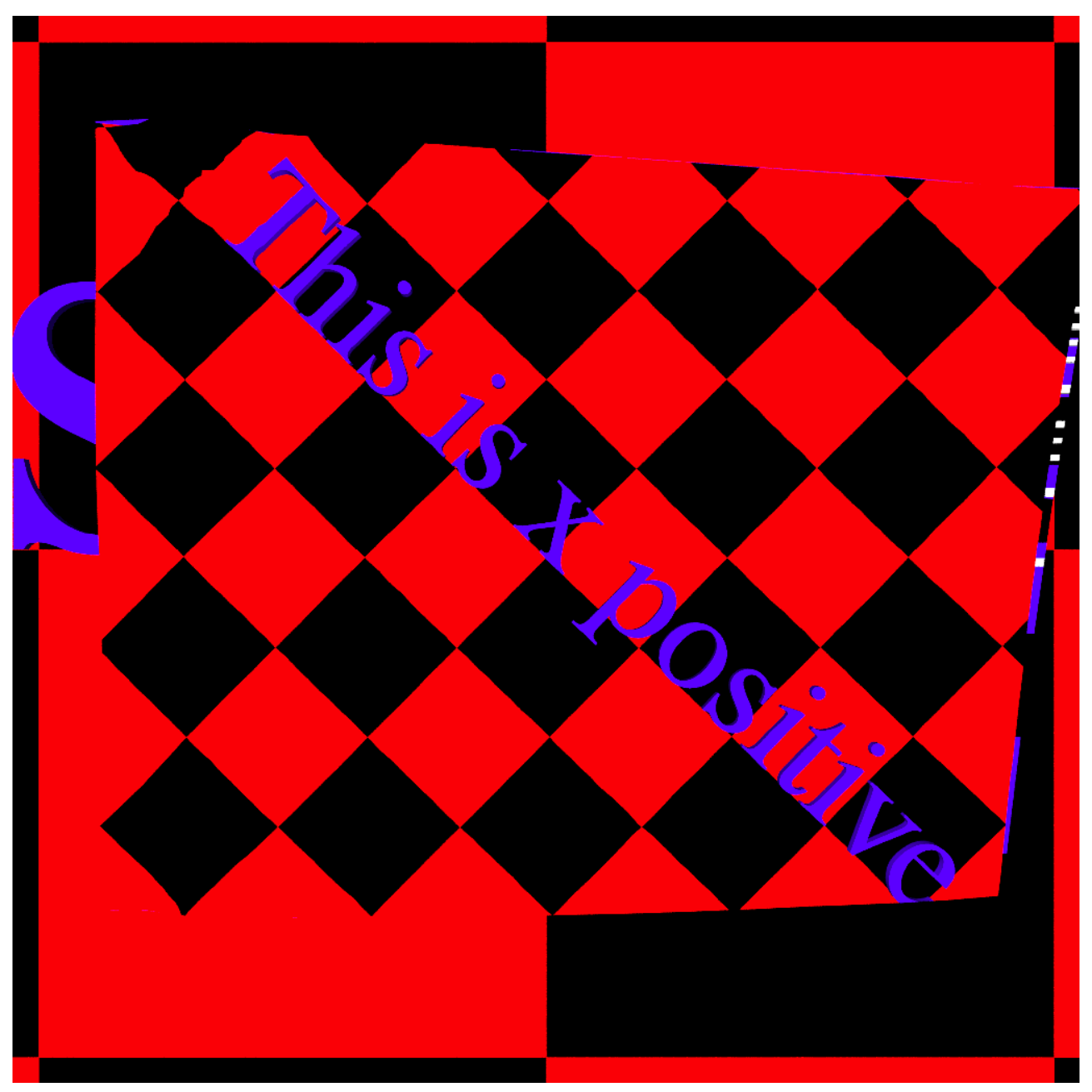

Fig. 7. A raytracing simulation of the mirror pair in Fig. 6. 


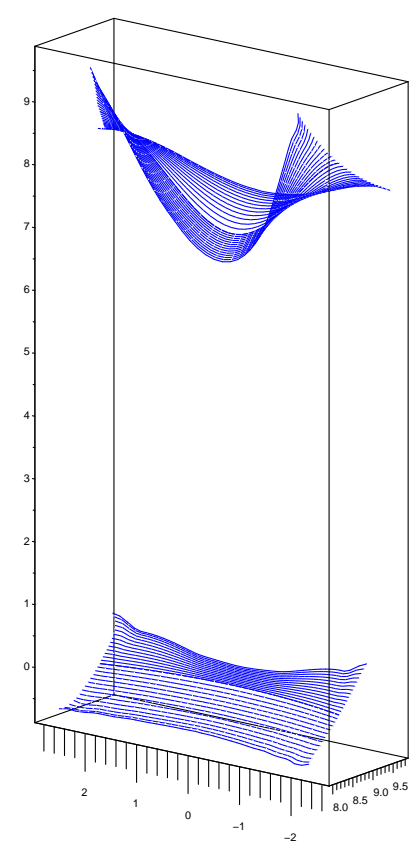

Fig. 8. A pair of mirrors that gives a panoramic view. 


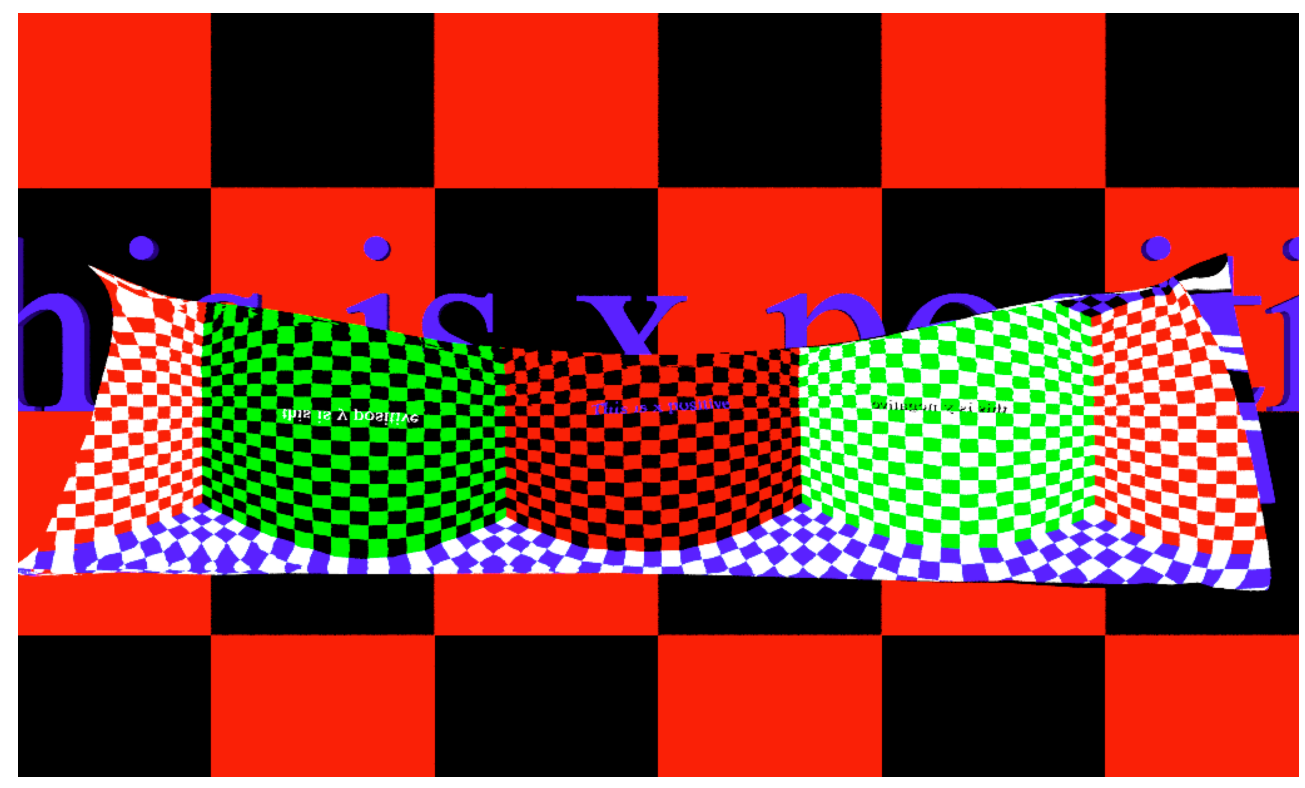

Fig. 9. A raytracing simulation of the mirror pair in Fig. 8. 


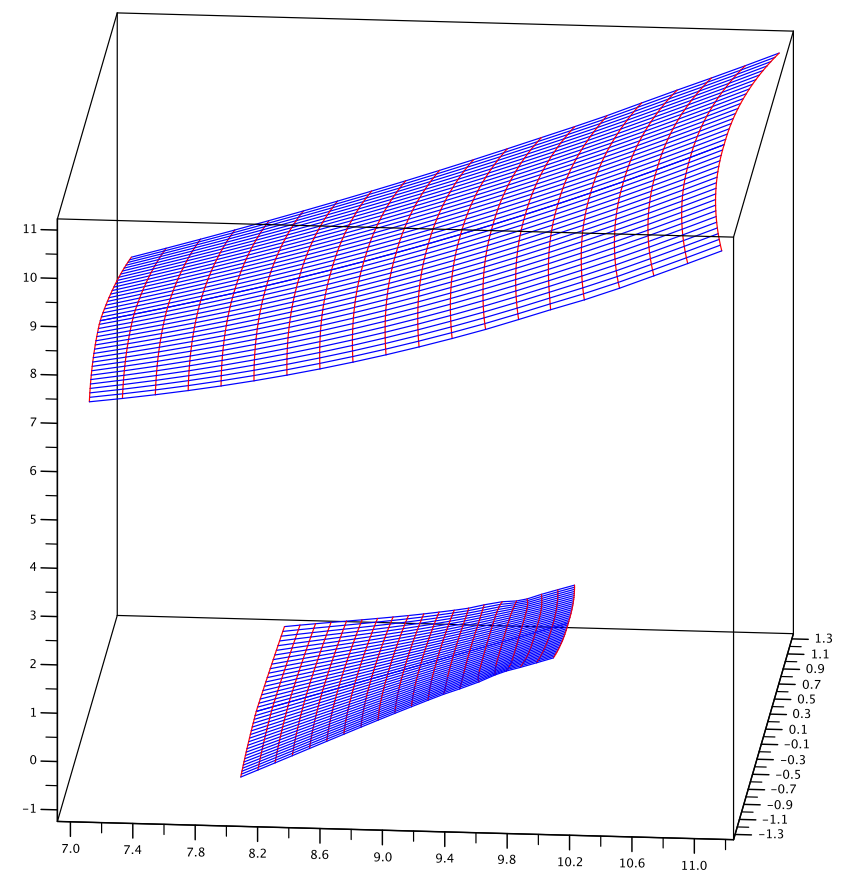

Fig. 10. The wide angle mirror pair generated by the "straight line vertical" initial data. Compare to Fig. 4. 


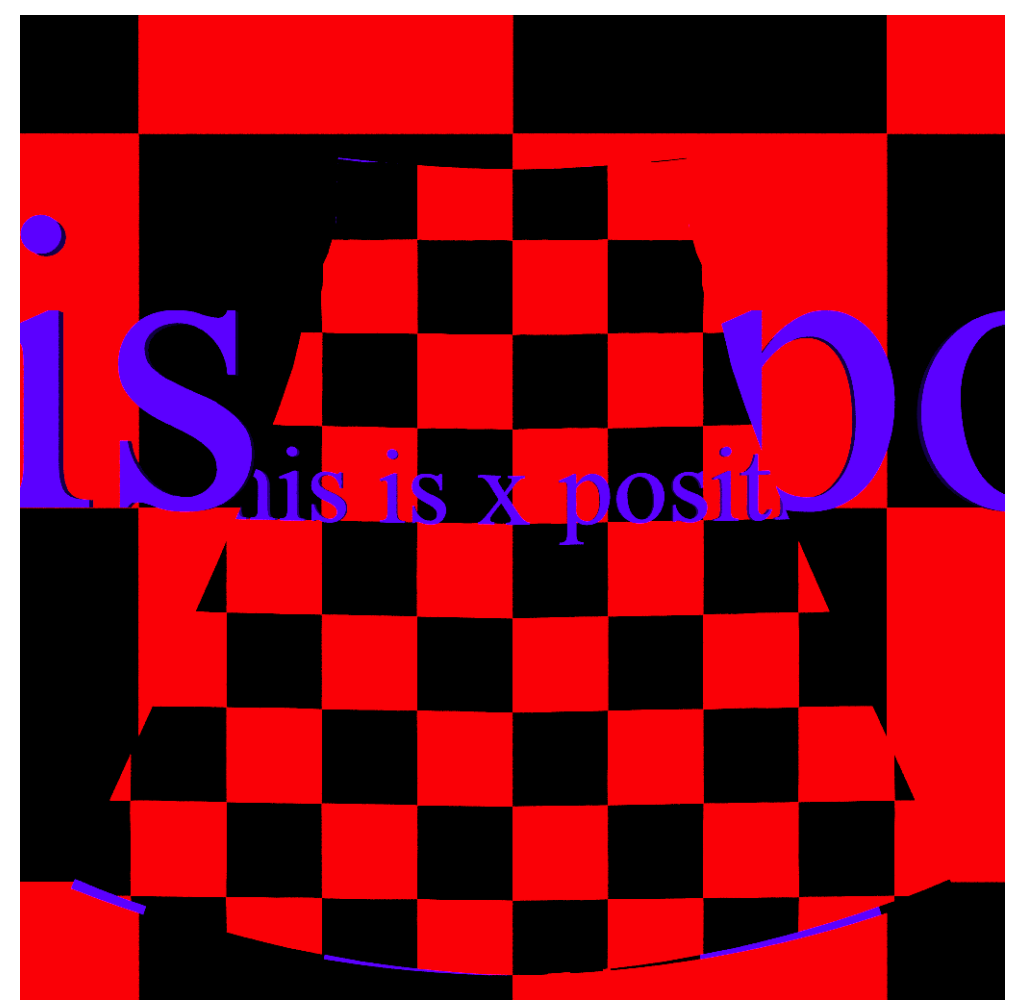

Fig. 11. A raytracing simulation of the mirror pair in Fig. 10. 